\title{
Typical Antipsychotics Increase Risk of Severe Exacerbation in Asthma Patients- A Nationwide Population-Based Cohort Study
}

\section{Chin-Wei Kuo}

National Cheng Kung University Hospital, National Cheng Kung University

\section{Szu-Chun Yang}

National Cheng Kung University Hospital, National Cheng Kung University

\section{Yu-Fen Shih}

National Cheng Kung University Hospital, National Cheng Kung University

\section{Xin-Min Liao}

National Cheng Kung University Hospital, National Cheng Kung University

Sheng-Hsiang Lin ( $\nabla$ shlin992@mail.ncku.edu.tw)

National Cheng Kung University

\section{Research Article}

Keywords: Asthma, Exacerbation, Antipsychotics

Posted Date: August 9th, 2021

DOl: https://doi.org/10.21203/rs.3.rs-778754/v1

License: (9) This work is licensed under a Creative Commons Attribution 4.0 International License. Read Full License 


\section{Abstract}

\section{Background:}

Severe asthma exacerbation reduces patients' life quality, results in visits to the emergency department (ED) and hospitalization, and incurs additional medical costs. Antipsychotics block receptors with bronchodilation function; however, the effects of antipsychotics use on severe asthma exacerbation are unknown. This study aimed to investigate the effects of antipsychotics on asthma-related ED visits and hospitalizations.

\section{Methods:}

This study used a case-crossover design. Using the 2003-2017 Taiwan National Health Insurance Reimbursement Database, we established a cohort of 18,657 adults with severe asthma exacerbation leading to ED visits or hospitalization. Univariate and multivariate conditional logistic regressions were conducted to explore the association of antipsychotics use with severe asthma exacerbation. Subgroup analyses of different classes, doses, receptor functions of antipsychotics and schizophrenia were also performed.

\section{Results:}

Antipsychotics use was associated with a higher risk of severe asthma exacerbation (adjusted odds ratio $(\mathrm{OR}): 1.27 ; 95 \%$ confidence interval $(\mathrm{Cl}): 1.05-1.54 ; P=0.013)$ compared with no use of antipsychotics. Use of typical antipsychotics increased the risk of severe asthma exacerbation (adjusted OR: 1.40, 95\% Cl: $1.10-1.79, P=0.007)$, whereas use of atypical antipsychotics did not. There was a dose-dependent effect of antipsychotics (test for trend: $P=0.025$ ). Antipsychotics that block the M2 muscarinic or D2 dopaminergic receptor were associated with an increased risk of severe asthma exacerbation (adjusted OR: $1.39,95 \% \mathrm{Cl}: 1.10-1.76, P=0.007$ and adjusted OR: $1.33,95 \% \mathrm{Cl}: 1.08-1.63, P=0.008$, respectively).

\section{Conclusions:}

Use of typical antipsychotics is associated with a dose-dependent increased risk of severe asthma exacerbation. Physicians should thus weight the risk and benefit of prescribing high-dose typical antipsychotics for asthma patients.

\section{Introduction}

Asthma is a prevalent airway disease with variable respiratory symptoms and airflow limitations.[1] It is the second most common chronic respiratory disease globally and accounts for $0.88 \%$ of all-cause mortality in 2017.[2] Exacerbation of asthma is defined as an episodic and progressive increase in asthma-associated respiratory symptoms, which impair patients' health-related quality of life.[3] Severe exacerbation of asthma leading to emergency department (ED) visits or hospitalization causes human 
productivity loss and incurs additional medical costs.[4] Moreover, it increases risk of future exacerbations.[5]

Antipsychotics are used in 3.5\% of Taiwan.[6] In addition to treating schizophrenic disorder, antipsychotics are administrated for a variety of psychiatric disorders, such as mood disorder, agitation, delirium, and insomnia.[7, 8] Off-label prescriptions of antipsychotics account for $40-75 \%$ of all prescriptions in adults. [9] Antipsychotics can be classified into typical and atypical ones based on their affinity to the D2 dopaminergic receptor and the serotonin 5-HT2A receptor, the side effects are different between the two groups of drugs because their affinity to different receptors. [10] In addition to dopaminergic receptor and serotonin receptors, antipsychotics are multipotent drugs that block several neurotransmitter receptors,[10] including the $\mathrm{M} 2$ muscarinic receptor, and $\beta_{2}$ adrenergic receptor.[11] The blocking of bronchodilation receptors, such as the $\beta_{2}$ adrenergic, may be associated with acute asthma exacerbation.[12] Crane et al. found a higher risk of asthma-related death and hospital readmission for antipsychotics users.[13] However, this case-control study only analyzed a small number of subjects receiving psychotropic drugs and the enrolled population were limited to people aged 5-45 years in New Zealand during 1981-1987. Most atypical antipsychotics drugs that are used clinically received approval from the Food and Drug Administration after the 1990s.[14] The risk of severe asthma exacerbation with the administration of atypical antipsychotics is still unclear. We hypothesized that the use of antipsychotics is associated with an increase of severe asthma exacerbation leading to ED visits or hospitalization. Using nationwide claims data, we conducted a case-crossover study to validate our hypothesis. We also tested the dose-dependent effect and performed subgroup analyses regarding different classes and receptor functions of antipsychotics.

\section{Methods}

\section{Study setting and design}

We performed this study using the 2003-2017 Taiwan National Health Insurance Reimbursement Database (NHIRD), which was derived from Taiwan Health and Welfare Data Science Center. The database includes the administrative data of 23 million people and covers $98 \%$ of residents in Taiwan. [15] This study used a case-crossover design. We compared the use of antipsychotics and other clinical factors during the period immediately prior to severe asthma exacerbation with that during an earlier control period. The Institutional Review Board of National Cheng Kung University Hospital approved this study before commencement (B-EX-109-026). Informed consent was waived because of the use of deidentified information.

\section{Definition of asthma patients and severe asthma exacerbation}

All asthma patients newly diagnosed between January 1, 2003, and December 31, 2016, were identified (Fig. 1A). We defined asthma patients as those who had more than one inpatient or two outpatient visits 
within 1 year using the International Classification of Diseases, Ninth Revision (ICD-9) diagnosis codes 493.xx or ICD-10-CM (Clinical Manifestation) codes J45.x.[15] The accuracy of diagnoses recorded in the NHIRD for asthma has been validated.[16] The first visit was defined as the day of asthma diagnosis. We excluded patients with a diagnosis of asthma between January 1,2000, and December 31, 2002, to ensure that all the cases were newly diagnosed. Patients with ICD-9 or ICD-10 codes for chronic obstructive pulmonary disease, chronic bronchiolitis, emphysema, and bronchiectasis, those younger than 18 years of age, and those who lacked sex information were also excluded. Every patient must had at least one asthma-related ED visit or hospitalization during the study period.

Severe asthma exacerbation was defined as an acute exacerbation of asthma resulting in ED visits or hospitalization in combination with the use of short-acting bronchodilators and systemic steroids. To avoid being confounded by previous severe exacerbation, we selected the first episode for analysis. ED visits and hospitalization within 180 days of the day of asthma diagnosis were excluded to avoid confounding from inadequate disease-control-related acute exacerbation at asthma diagnosis.

\section{Case and control periods}

Based on previous studies and the elimination half-lives of antipsychotics (Supplementary Table 1),[17, 18] we defined the case period as 1 to 14 days prior to severe asthma exacerbation and the control period as 75 to 88 days prior to severe asthma exacerbation (Fig. 1B). These definitions ensured washout of the drug effects. Patients' co-morbidities during the 180 days before the case period were compared with those during the 180 days before the control period to derive the odds ratios (ORs). The use of other medications during the case period were compared with that during the control period. The ICD codes of comorbidities and medications are listed in Supplementary Table 2 and Supplementary Table 3.

\section{Statistical analysis}

In order to accommodate the case-crossover design, we used conditional logistic regression analyses to deal with matched pair data. We first conducted a univariable analysis to derive the crude OR of each covariate in the case period versus the control period. Covariates with significant crude ORs $(P<0.05)$ in the univariable analysis were used the multivariable analysis to obtain the adjusted ORs. To test the robustness of the results, we performed several sensitivity analyses. First, we categorized asthma patients by use of typical antipsychotics, atypical antipsychotics, or both. Second, we examined the dosedependent effect of antipsychotics by dividing the study cohort into low-dose and medium- to high-dose groups. We defined both groups based on the defined daily dose (DDD).[19] A low dose denoted a dose of less than or equal to $0.25 \mathrm{DDD}$ and a medium to high dose denoted a dose of more than 0.25 DDD.[17] Cochran-Armitage trend test was used to confirm whether the antipsychotics have a dose dependent effect on severe asthma exacerbation. Third, because of schizophrenia is the main indication of antipsychotics. We performed the analysis after excluding enrolled subjects with schizophrenia for avoiding the confounding effect of schizophrenia. Forth, we classified each antipsychotic by its antagonism property on different receptors with the bronchial relaxation function, which include the $\beta 2$ adrenergic, M2 muscarinic, D1 dopaminergic, and D2 dopaminergic receptors.[20-23] We defined Ki 
value $<100$ as higher affinity and Ki value $>100$ as lower affinity (Supplementary Table 4).[11] All analyses were performed using SAS software (Version 9.4; SAS Institute, Cary NC, USA) and $P$ values were based on two-tail tests.

\section{Results}

A total of 18,657 patients from 2003 to 2016 with severe asthma exacerbation resulting in ED visits or hospitalization in combination with the use of short-acting bronchodilators and systemic steroids were included for analysis. Demographic characteristics, comorbidities, and drugs exposure during the case and control periods are shown on Table 1. Most of the patients were female. The mean age was 47.7 years on the event day. Enrolled patients were more likely to have more diagnoses of comorbidities, schizophrenia, pneumonia, and acute upper airway infection in the case period. Moreover, enrolled patients were more likely to use antiplatelet, $\beta$-blockers, NSAIDs, antidepressants, antipsychotics, anticholinergic agents, antihistamine, inhaled bronchodilators, and oral bronchodilators in the case period than in the control period (Table 1). 
Table 1

Characteristics of patients in the case and control periods.

\begin{tabular}{|c|c|c|c|c|}
\hline \multirow{3}{*}{ Characteristics } & \multicolumn{2}{|l|}{ Number (\%) } & \multicolumn{2}{|l|}{$\begin{array}{l}\text { Conditional logistic } \\
\text { regression }\end{array}$} \\
\hline & $\begin{array}{l}\text { Case } \\
\text { period }\end{array}$ & $\begin{array}{l}\text { Control } \\
\text { period }\end{array}$ & & $P$ value \\
\hline & $(n=18657)$ & $(n=18657)$ & $(95 \% \mathrm{Cl})$ & \\
\hline Male & $\begin{array}{l}5577 \\
(29.9)\end{array}$ & $5577(29.9)$ & - & - \\
\hline Age, mean (SD) & $47.7(17.9)$ & $47.5(17.7)$ & & \\
\hline \multicolumn{5}{|l|}{ Comorbidities: } \\
\hline Heart failure & $395(2.1)$ & $349(1.9)$ & $2.18(1.49-3.18)$ & $<0.001$ \\
\hline Ischemic heart disease & $931(5.0)$ & $873(4.7)$ & $1.62(1.25-2.10)$ & $<0.001$ \\
\hline Stroke & $530(2.8)$ & $498(2.7)$ & $1.62(1.14-2.28)$ & 0.007 \\
\hline GERD & $762(4.1)$ & $671(3.6)$ & $1.65(1.34-2.04)$ & $<0.001$ \\
\hline Obesity & $77(0.4)$ & $49(0.3)$ & $4.11(1.98-8.52)$ & $<0.001$ \\
\hline Rhinosinusitis & $\begin{array}{l}3393 \\
(18.2)\end{array}$ & $3125(16.8)$ & $1.44(1.30-1.60)$ & $<0.001$ \\
\hline Obstructive sleep apnea & $51(0.3)$ & $44(0.2)$ & $1.64(0.77-3.46)$ & 0.198 \\
\hline \multicolumn{5}{|l|}{ Psychiatric disorder } \\
\hline Anxiety & $166(0.9)$ & $159(0.9)$ & $1.21(0.76-1.92)$ & 0.415 \\
\hline Bipolar disorder & $154(0.8)$ & $146(0.8)$ & $1.62(0.81-3.23)$ & 0.174 \\
\hline Depression & $747(4.0)$ & $741(4.0)$ & $1.08(0.79-1.47)$ & 0.633 \\
\hline Schizophrenia & $148(0.8)$ & $136(0.7)$ & $5.00(1.45-17.27)$ & 0.011 \\
\hline \multicolumn{5}{|l|}{ Respiratory infection } \\
\hline Pneumonia & $726(3.9)$ & $533(2.9)$ & $2.44(2.00-2.98)$ & $<0.001$ \\
\hline Acute upper airway infection & $\begin{array}{l}8308 \\
(44.5)\end{array}$ & $7140(38.3)$ & $2.00(1.86-2.14)$ & $<0.001$ \\
\hline \multicolumn{5}{|l|}{ Medications: } \\
\hline Cardiovascular drugs & & & & \\
\hline
\end{tabular}

GERD: gastroesophageal reflux disease; ICS: inhaled corticosteroid, LABA: long-acting $\beta$-agonist; LAMA: long-acting muscarinic antagonist; NSAID: non-steroidal anti-inflammatory drug; OR: odds ratio; SABA: short-acting $\beta$-agonist; SAMA: short-acti 


\begin{tabular}{|c|c|c|c|c|}
\hline & \multicolumn{2}{|c|}{ Number (\%) } & \multicolumn{2}{|l|}{$\begin{array}{l}\text { Conditional logistic } \\
\text { regression }\end{array}$} \\
\hline Antiplatelet & $1184(6.4)$ & $1019(5.5)$ & $1.52(1.32-1.75)$ & $<0.001$ \\
\hline Cardioselective $\beta$-blockers & $716(3.8)$ & $660(3.5)$ & $1.24(1.04-1.47)$ & 0.015 \\
\hline Non-selective $\beta$-blockers & $676(3.6)$ & $553(3.0)$ & $1.52(1.29-1.78)$ & $<0.001$ \\
\hline Statins & $909(4.9)$ & $883(4.7)$ & $1.08(0.93-1.25)$ & 0.324 \\
\hline \multicolumn{5}{|l|}{ Anti-inflammation } \\
\hline Colchicine & $138(0.7)$ & $147(0.8)$ & $0.90(0.67-1.22)$ & 0.492 \\
\hline NSAIDs & $\begin{array}{l}5175 \\
(27.7)\end{array}$ & $3098(16.6)$ & $2.32(2.19-2.47)$ & $<0.001$ \\
\hline Metformin & $753(4.0)$ & $721(3.9)$ & $1.15(0.96-1.39)$ & 0.133 \\
\hline \multicolumn{5}{|l|}{ Psychoactive drugs } \\
\hline Antidepressants & $957(5.1)$ & $857(4.6)$ & $1.39(1.19-1.64)$ & $<0.001$ \\
\hline Lithium & $16(0.1)$ & $13(0.1)$ & $2.00(0.50-8.00)$ & 0.327 \\
\hline Antipsychotics & $713(3.8)$ & $601(3.2)$ & $1.48(1.25-1.74)$ & $<0.001$ \\
\hline \multicolumn{5}{|l|}{ Anticholinergic agents } \\
\hline Bladder & $116(0.6)$ & $93(0.5)$ & $1.43(1.01-2.04)$ & 0.044 \\
\hline Gastrointestinal tract & $1442(7.7)$ & $888(4.8)$ & $1.86(1.69-2.05)$ & $<0.001$ \\
\hline Parkinsonism & $107(0.6)$ & $100(0.5)$ & $1.28(0.76-2.16)$ & 0.358 \\
\hline \multicolumn{5}{|l|}{ Antihistamine } \\
\hline First generation & $\begin{array}{l}3234 \\
(17.3)\end{array}$ & $1817(9.7)$ & $2.26(2.11-2.43)$ & $<0.001$ \\
\hline Second/third generation & $\begin{array}{l}4372 \\
(23.4)\end{array}$ & $2615(14.0)$ & $2.27(2.13-2.42)$ & $<0.001$ \\
\hline \multicolumn{5}{|l|}{ Inhaled bronchodilators } \\
\hline SABA & $\begin{array}{l}2615 \\
(14.0)\end{array}$ & $1087(5.8)$ & $3.25(2.98-3.54)$ & $<0.001$ \\
\hline SAMA & $305(1.6)$ & $60(0.3)$ & $6.56(4.78-9.01)$ & $<0.001$ \\
\hline SABA + SAMA & $154(0.8)$ & $79(0.4)$ & $2.50(1.80-3.47)$ & $<0.001$ \\
\hline
\end{tabular}

GERD: gastroesophageal reflux disease; ICS: inhaled corticosteroid, LABA: long-acting $\beta$-agonist; LAMA: long-acting muscarinic antagonist; NSAID: non-steroidal anti-inflammatory drug; OR: odds ratio; SABA: short-acting $\beta$-agonist; SAMA: short-acti 


\begin{tabular}{|c|c|c|c|c|}
\hline & \multicolumn{2}{|l|}{ Number (\%) } & \multicolumn{2}{|l|}{$\begin{array}{l}\text { Conditional logistic } \\
\text { regression }\end{array}$} \\
\hline LABA & $3(0.02)$ & $0(0.0)$ & - & - \\
\hline LAMA & $37(0.2)$ & $30(0.2)$ & $1.44(0.76-2.72)$ & 0.265 \\
\hline$L A B A+I C S$ & $1575(8.4)$ & $1217(6.5)$ & $1.55(1.41-1.72)$ & $<0.001$ \\
\hline$L A B A+L A M A+I C S$ & $0(0.0)$ & $0(0.0)$ & - & - \\
\hline ICS & $381(2.0)$ & $283(1.5)$ & $1.50(1.25-1.80)$ & $<0.001$ \\
\hline \multicolumn{5}{|l|}{ Oral bronchodilators } \\
\hline Xanthium & $\begin{array}{l}3844 \\
(20.6)\end{array}$ & $1771(9.5)$ & $3.40(3.15-3.66)$ & $<0.001$ \\
\hline $\begin{array}{l}\text { Leukotriene receptor } \\
\text { antagonists }\end{array}$ & $550(3.0)$ & $404(2.2)$ & $1.78(1.49-2.12)$ & $<0.001$ \\
\hline \multicolumn{5}{|c|}{$\begin{array}{l}\text { GERD: gastroesophageal reflux disease; ICS: inhaled corticosteroid, LABA: long-acting } \beta \text {-agonist; } \\
\text { LAMA: long-acting muscarinic antagonist; NSAID: non-steroidal anti-inflammatory drug; OR: odds } \\
\text { ratio; SABA: short-acting } \beta \text {-agonist; SAMA: short-acti }\end{array}$} \\
\hline
\end{tabular}

Table 2 shows the results of conditional logistic regression for the risk of severe asthma exacerbation associated with any use of antipsychotics. There were $713(3.8 \%)$ patients with any use of antipsychotics during the case period and 601 (3.2\%) patients during the control period. The adjusted OR was 1.27 (95\% confidence interval $(\mathrm{Cl}), 1.05-1.54 ; P=0.013)$. The adjusted ORs of all covariates in multivariable conditional logistic regression are shown in Supplementary Table 5. Schizophrenia increased the risk of severe asthma exacerbation (adjusted OR: 5.46; 95\% Cl: 1.48-20.18; $P=0.011$ ). After excluding patients with schizophrenia, any use of antipsychotics still increased the risk of severe asthma exacerbation (adjusted OR: 1.29; 95\% Cl: 1.07-1.57, P=0.009) (Table 3). 
Table 2

Risk of severe asthma exacerbation by different classes of antipsychotics and doses

Number (\%)

\begin{tabular}{|c|c|c|c|c|c|c|}
\hline Characteristics & $\begin{array}{l}\text { Case } \\
\text { period } \\
\\
(n= \\
18657)\end{array}$ & $\begin{array}{l}\text { Control } \\
\text { period } \\
(n= \\
18657)\end{array}$ & $\begin{array}{l}\text { Crude OR } \\
(95 \% \mathrm{Cl})\end{array}$ & $\begin{array}{l}P \\
\text { value }\end{array}$ & $\begin{array}{l}\text { Adjusted } \\
\text { OR }^{\text {a }} \\
(95 \% \mathrm{Cl})\end{array}$ & $\begin{array}{l}P \\
\text { value }\end{array}$ \\
\hline $\begin{array}{l}\text { No use of } \\
\text { antipsychotics }\end{array}$ & $\begin{array}{l}17944 \\
(96.18)\end{array}$ & $\begin{array}{l}18056 \\
(96.78)\end{array}$ & Ref. & & Ref. & \\
\hline $\begin{array}{l}\text { Any use of } \\
\text { antipsychotics }\end{array}$ & $\begin{array}{l}713 \\
(3.8)\end{array}$ & $\begin{array}{l}601 \\
(3.2)\end{array}$ & $\begin{array}{l}1.48 \\
(1.25- \\
1.74)\end{array}$ & $\begin{array}{l}< \\
0.001\end{array}$ & $\begin{array}{l}1.27 \\
(1.05- \\
1.54)\end{array}$ & 0.013 \\
\hline
\end{tabular}

Antipsychotics

class $^{\mathrm{b}}$

\begin{tabular}{|c|c|c|c|c|c|c|}
\hline Typical only & $\begin{array}{l}269 \\
(1.44)\end{array}$ & $\begin{array}{l}193 \\
(1.03)\end{array}$ & $\begin{array}{l}1.63 \\
(1.31- \\
2.04)\end{array}$ & $\begin{array}{l}<.001 \\
0.001\end{array}$ & $\begin{array}{l}1.40 \\
(1.10- \\
1.79)\end{array}$ & 0.007 \\
\hline Atypical only & $\begin{array}{l}411 \\
(2.20)\end{array}$ & $\begin{array}{l}390 \\
(2.09)\end{array}$ & $\begin{array}{l}1.25 \\
(0.98- \\
1.59)\end{array}$ & 0.072 & $\begin{array}{l}1.10 \\
(0.84- \\
1.44)\end{array}$ & 0.481 \\
\hline Both & $\begin{array}{l}33 \\
(0.18)\end{array}$ & $\begin{array}{l}18 \\
(0.10)\end{array}$ & $\begin{array}{l}3.03 \\
(1.40- \\
6.57)\end{array}$ & 0.005 & $\begin{array}{l}2.47 \\
(1.09- \\
5.62)\end{array}$ & 0.031 \\
\hline
\end{tabular}

Dose

\begin{tabular}{|c|c|c|c|c|c|c|}
\hline $\operatorname{Low}(\leq 0.25 \mathrm{DDD})$ & $\begin{array}{l}604 \\
(3.24)\end{array}$ & $\begin{array}{l}504 \\
(2.70)\end{array}$ & $\begin{array}{l}1.47 \\
(1.24- \\
1.74)\end{array}$ & $<.001$ & $\begin{array}{l}1.27 \\
(1.05- \\
1.53)\end{array}$ & 0.013 \\
\hline $\begin{array}{l}\text { Medium to high (> } \\
0.25 \text { DDD) }\end{array}$ & $\begin{array}{l}109 \\
(0.58)\end{array}$ & $\begin{array}{l}97 \\
(0.52)\end{array}$ & $\begin{array}{l}1.57 \\
(1.11- \\
2.21)\end{array}$ & 0.011 & $\begin{array}{l}1.47 \\
(1.01- \\
2.15)\end{array}$ & 0.045 \\
\hline
\end{tabular}

DDD: defined daily dose; OR: odds ratio.

a Adjusted for heart failure, ischemic heart disease, stroke, gastroesophageal reflux disease, obesity disorder, rhinosinusitis, psychiatric disorder, schizophrenia, pneumonia, acute upper airway infection, antiplatelet agents, cardioselective $\beta$-blocker, non-selective $\beta$-blocker, NSAID, anti-psychotics, antidepressants, bladder anticholinergic agents, gastrointestinal tract anticholinergic agents, first generation anti-histamine, second/third generation anti-histamine, short-acting beta-agonist, shortacting muscarinic antagonist, short-acting beta-agonist plus short-acting muscarinic antagonist, longacting beta-agonist plus long-acting beta-agonist, long-acting beta-agonist, xanthine inhibitor, leukotriene receptor antagonist.

b See Supplementary Table 1. 


\section{Number (\%)}

Conditional logistic regression

Dose (typical

only)

\begin{tabular}{|c|c|c|c|c|c|c|c|}
\hline $\operatorname{Low}(\leq 0.25 \mathrm{DDD})$ & $\begin{array}{l}255 \\
(1.37)\end{array}$ & $\begin{array}{l}184 \\
(0.99)\end{array}$ & $\begin{array}{l}1.61 \\
(1.28- \\
2.02)\end{array}$ & $<.001$ & $\begin{array}{l}1.37 \\
(1.06- \\
1.76)\end{array}$ & 0.015 & 0.006 \\
\hline $\begin{array}{l}\text { Medium to high (> } \\
0.25 \text { DDD) }\end{array}$ & $\begin{array}{l}14 \\
(0.08)\end{array}$ & $9(0.05)$ & $\begin{array}{l}2.85 \\
(0.96- \\
8.50)\end{array}$ & 0.060 & $\begin{array}{l}4.50 \\
(1.36- \\
14.94)\end{array}$ & 0.014 & \\
\hline
\end{tabular}

Dose (atypical

only)

Low ( $\leq 0.25$ DDD)

326

(1.75)

311

(1.67)

1.27

(0.99-

0.066

1.11

(0.84-

0.479

0.652

1.63)

1.46)

Medium to high ( $>\quad 85$

(0.46)

79

$(0.42)$

1.36

(0.91-

0.134

1.21

(0.78-

0.385

2.03)

1.88)

DDD: defined daily dose; OR: odds ratio.

${ }^{a}$ Adjusted for heart failure, ischemic heart disease, stroke, gastroesophageal reflux disease, obesity disorder, rhinosinusitis, psychiatric disorder, schizophrenia, pneumonia, acute upper airway infection, antiplatelet agents, cardioselective $\beta$-blocker, non-selective $\beta$-blocker, NSAID, anti-psychotics, antidepressants, bladder anticholinergic agents, gastrointestinal tract anticholinergic agents, first generation anti-histamine, second/third generation anti-histamine, short-acting beta-agonist, shortacting muscarinic antagonist, short-acting beta-agonist plus short-acting muscarinic antagonist, longacting beta-agonist plus long-acting beta-agonist, long-acting beta-agonist, xanthine inhibitor, leukotriene receptor antagonist.

\section{b See Supplementary Table 1.}


Table 3

Risk of severe asthma exacerbation by different classes of antipsychotics and doses after excluding schizophrenia patients

\begin{tabular}{|c|c|c|c|c|c|c|c|}
\hline \multirow[b]{2}{*}{ Characteristics } & \multicolumn{2}{|l|}{ No. (\%) } & \multicolumn{5}{|c|}{ Conditional logistic regression } \\
\hline & $\begin{array}{l}\text { Case } \\
\text { period }\end{array}$ & $\begin{array}{l}\text { Control } \\
\text { period }\end{array}$ & $\begin{array}{l}\text { Crude OR } \\
(95 \% \mathrm{Cl})\end{array}$ & $\begin{array}{l}\mathrm{P} \\
\text { value }\end{array}$ & $\begin{array}{l}\text { Adjusted } \\
\text { OR }^{a} \\
(95 \% \mathrm{Cl})\end{array}$ & $\begin{array}{l}\mathrm{P} \\
\text { value }\end{array}$ & $\begin{array}{l}\text { P for } \\
\text { trend }\end{array}$ \\
\hline $\begin{array}{l}\text { No use of } \\
\text { antipsychotics }\end{array}$ & $\begin{array}{l}17900 \\
(96.73)\end{array}$ & $\begin{array}{l}18008 \\
(97.31)\end{array}$ & Ref. & & Ref. & & \\
\hline $\begin{array}{l}\text { Any use of anti- } \\
\text { psychotics }\end{array}$ & $\begin{array}{l}606 \\
(3.3)\end{array}$ & $\begin{array}{l}498 \\
(2.7)\end{array}$ & $\begin{array}{l}1.49 \\
(1.26- \\
1.77)\end{array}$ & $\begin{array}{l}< \\
0.001\end{array}$ & $\begin{array}{l}1.29 \\
(1.07- \\
1.57)\end{array}$ & 0.009 & \\
\hline \multicolumn{8}{|l|}{$\begin{array}{l}\text { Antipsychotics } \\
\text { class }^{b}\end{array}$} \\
\hline Typical only & $\begin{array}{l}260 \\
(1.40)\end{array}$ & $\begin{array}{l}186 \\
(1.01)\end{array}$ & $\begin{array}{l}1.62 \\
(1.29- \\
2.03)\end{array}$ & $<0.001$ & $\begin{array}{l}1.37 \\
(1.07- \\
1.76)\end{array}$ & 0.012 & \\
\hline Atypical only & $\begin{array}{l}326 \\
(1.76)\end{array}$ & $\begin{array}{l}298 \\
(1.61)\end{array}$ & $\begin{array}{l}1.32 \\
(1.01- \\
1.71)\end{array}$ & 0.039 & $\begin{array}{l}1.17 \\
(0.88- \\
1.56)\end{array}$ & 0.293 & \\
\hline Both & $\begin{array}{l}20 \\
(0.11)\end{array}$ & $\begin{array}{l}14 \\
(0.08)\end{array}$ & $\begin{array}{l}1.87 \\
(0.81- \\
4.30)\end{array}$ & 0.142 & $\begin{array}{l}1.63 \\
(0.67- \\
3.97)\end{array}$ & 0.285 & \\
\hline \multicolumn{8}{|l|}{ Dose } \\
\hline $\operatorname{Low}(\leq 0.25 \mathrm{DDD})$ & $\begin{array}{l}535 \\
(2.89)\end{array}$ & $\begin{array}{l}441 \\
(2.38)\end{array}$ & $\begin{array}{l}1.48 \\
(1.25- \\
1.76)\end{array}$ & $<0.001$ & $\begin{array}{l}1.28 \\
(1.05- \\
1.55)\end{array}$ & 0.013 & 0.018 \\
\hline $\begin{array}{l}\text { Medium to high } \\
(>0.25 \mathrm{DDD})\end{array}$ & $\begin{array}{l}71 \\
(0.38)\end{array}$ & $\begin{array}{l}57 \\
(0.31)\end{array}$ & $\begin{array}{l}1.81 \\
(1.17- \\
2.78)\end{array}$ & 0.007 & $\begin{array}{l}1.66 \\
(1.04- \\
2.63)\end{array}$ & 0.034 & \\
\hline
\end{tabular}

DDD: defined daily dose, OR: odds ratio

a Adjusted for heart failure, ischemic heart disease, stroke, gastroesophageal reflux disease, obesity disorder, rhinosinusitis, psychiatric disorder, schizophrenia, pneumonia, acute upper airway infection, antiplatelet agents, cardioselective $\beta$-blocker, non-selective $\beta$-blocker, NSAID, anti-psychotics, antidepressants, bladder anticholinergic agents, gastrointestinal tract anticholinergic agents, first generation anti-histamine, second/third generation anti-histamine, short-acting beta-agonist, shortacting muscarinic antagonist, short-acting beta-agonist plus short-acting muscarinic antagonist, longacting beta-agonist plus long-acting beta-agonist, long-acting beta-agonist, xanthine inhibitor, leukotriene receptor antagonist.

b See Supplementary Table 1. 


\title{
No. (\%)
}

\section{Conditional logistic regression}

Dose (typical

only)

\begin{tabular}{|c|c|c|c|c|c|c|c|}
\hline $\operatorname{Low}(\leq 0.25 \mathrm{DDD})$ & $\begin{array}{l}249 \\
(1.35)\end{array}$ & $\begin{array}{l}181 \\
(0.98)\end{array}$ & $\begin{array}{l}1.60 \\
(1.27- \\
2.01)\end{array}$ & $<.001$ & $\begin{array}{l}1.37 \\
(1.06- \\
1.76)\end{array}$ & 0.015 & 0.006 \\
\hline $\begin{array}{l}\text { Medium to high } \\
(>0.25 \mathrm{DDD})\end{array}$ & $\begin{array}{l}11 \\
(0.06)\end{array}$ & $5(0.03)$ & $\begin{array}{l}4.30 \\
(1.15- \\
16.15)\end{array}$ & 0.031 & $\begin{array}{l}5.74 \\
(1.33- \\
24.69)\end{array}$ & 0.019 & \\
\hline
\end{tabular}

Dose (atypical

only)

\begin{tabular}{|c|c|c|c|c|c|c|}
\hline Low $(\leq 0.25 \mathrm{DDD})$ & $\begin{array}{l}271 \\
(1.46)\end{array}$ & $\begin{array}{l}252 \\
(1.36)\end{array}$ & $\begin{array}{l}1.30 \\
(0.99- \\
1.70)\end{array}$ & 0.057 & $\begin{array}{l}1.15 \\
(0.85- \\
1.55)\end{array}$ & 0.364 \\
\hline
\end{tabular}

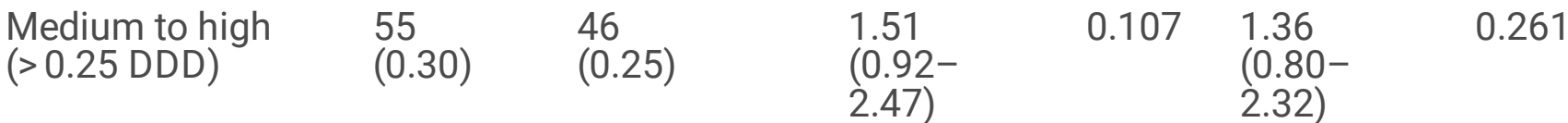

DDD: defined daily dose, OR: odds ratio

\begin{abstract}
a Adjusted for heart failure, ischemic heart disease, stroke, gastroesophageal reflux disease, obesity disorder, rhinosinusitis, psychiatric disorder, schizophrenia, pneumonia, acute upper airway infection, antiplatelet agents, cardioselective $\beta$-blocker, non-selective $\beta$-blocker, NSAID, anti-psychotics, antidepressants, bladder anticholinergic agents, gastrointestinal tract anticholinergic agents, first generation anti-histamine, second/third generation anti-histamine, short-acting beta-agonist, shortacting muscarinic antagonist, short-acting beta-agonist plus short-acting muscarinic antagonist, longacting beta-agonist plus long-acting beta-agonist, long-acting beta-agonist, xanthine inhibitor, leukotriene receptor antagonist.
\end{abstract}

\section{${ }^{b}$ See Supplementary Table 1.}

The risk of severe asthma exacerbation by different classes of antipsychotics and doses is shown on lower part of Table 2. Use of typical antipsychotics was associated with an increased risk of severe asthma exacerbation (adjusted OR: $1.40,95 \% \mathrm{Cl}: 1.10-1.79, P=0.007$. In contrast, use of atypical antipsychotics did not increase the risk (adjusted OR: 1.10, 95\% Cl: $0.84-1.44, P=0.481$ ). Simultaneously use both typical and atypical antipsychotics had higher risk of asthma exacerbation than use one of them (adjusted OR: 2.47, 95\% Cl: 1.09-5.62, $P=0.031$ ). A dose-dependent effect was also found in the use of any class of antipsychotics. The effect was obvious for the use of typical antipsychotics but not statistically significant for atypical antipsychotics (in the test for trend, any class of antipsychotics: $P=$ 0.025 , typical antipsychotics: $P=0.006$; atypical antipsychotics: $P=0.652)$. After excluding patients with schizophrenia, the result of analysis is similar to the result of analysis for all enrolled patients. Use of typical antipsychotics was still associated with increased risk of severe asthma exacerbation with dosedependent effect, but use of atypical antipsychotics was not (for typical antipsychotics: adjusted OR: 
1.37, $95 \% \mathrm{Cl}: 1.07-1.76, P=0.012$; for atypical antipsychotics: adjusted OR: $1.17,95 \% \mathrm{Cl}: 0.88-1.56, P=$ 0.293) (Table 3).

Table 4 shows the risk of severe asthma exacerbation stratified by different receptor functions of antipsychotics. Agents that block the M2 muscarinic receptor and the D2 dopaminergic receptor are associated with increased risk of severe asthma exacerbation (adjusted OR: 1.39, 95\% Cl: 1.10-1.76, $P=$ 0.007 and adjusted OR: $1.33,95 \% \mathrm{Cl}: 1.08-1.63, P=0.008$, respectively).

Table 4

Risk of severe asthma exacerbation of antipsychotics with high blocking affinity of receptor

\begin{tabular}{|c|c|c|c|c|c|c|}
\hline \multirow[b]{2}{*}{ Characteristics } & \multicolumn{2}{|c|}{ Number (\%) } & \multicolumn{4}{|c|}{ Conditional logistic regression } \\
\hline & $\begin{array}{l}\text { Case } \\
\text { period }\end{array}$ & $\begin{array}{l}\text { Control } \\
\text { period }\end{array}$ & $\begin{array}{l}\text { Crude OR } \\
(95 \% \mathrm{Cl})\end{array}$ & $\begin{array}{l}P \\
\text { value }\end{array}$ & $\begin{array}{l}\text { Adjusted } \\
\text { OR }^{a} \\
(95 \% \mathrm{Cl})\end{array}$ & $\begin{array}{l}P \\
\text { value }\end{array}$ \\
\hline No antipsychotics use & 17927 & 18037 & Ref. & & Ref. & \\
\hline \multicolumn{7}{|l|}{$\begin{array}{l}\text { Antipsychotics with high } \\
\text { blocking affinity b,c }\end{array}$} \\
\hline$\beta 2$ adrenergic receptor & $\begin{array}{l}302 \\
(1.7)\end{array}$ & $\begin{array}{l}276 \\
(1.5)\end{array}$ & $\begin{array}{l}1.37 \\
(0.98- \\
1.92)\end{array}$ & 0.064 & $\begin{array}{l}1.25 \\
(0.87- \\
1.81)\end{array}$ & 0.227 \\
\hline M2 muscarinic receptor & $\begin{array}{l}442 \\
(2.4)\end{array}$ & $\begin{array}{l}356 \\
(1.9)\end{array}$ & $\begin{array}{l}1.60 \\
(1.29- \\
1.99)\end{array}$ & $\dot{0} 001$ & $\begin{array}{l}1.39 \\
(1.10- \\
1.76)\end{array}$ & 0.007 \\
\hline D1 dopaminergic receptor & $\begin{array}{l}391 \\
(2.1)\end{array}$ & $\begin{array}{l}357 \\
(1.9)\end{array}$ & $\begin{array}{l}1.34 \\
(1.04- \\
1.73)\end{array}$ & 0.025 & $\begin{array}{l}1.17 \\
(0.88- \\
1.55)\end{array}$ & 0.283 \\
\hline D2 dopaminergic receptor & $\begin{array}{l}535 \\
(2.9)\end{array}$ & $\begin{array}{l}440 \\
(2.4)\end{array}$ & $\begin{array}{l}1.52 \\
(1.26- \\
1.84)\end{array}$ & $<0.001$ & $\begin{array}{l}1.33 \\
(1.08- \\
1.63)\end{array}$ & 0.008 \\
\hline \multicolumn{7}{|c|}{$\begin{array}{l}\text { a Adjusted for heart failure, ischemic heart disease, stroke, gastroesophageal reflux disease, obesity } \\
\text { disorder, rhinosinusitis, psychiatric disorder, schizophrenia, pneumonia, acute upper airway infection, } \\
\text { antiplatelet agents, cardioselective } \beta \text {-blocker, non-selective } \beta \text {-blocker, NSAID, anti-psychotics, anti- } \\
\text { depressants, bladder anticholinergic agents, gastrointestinal tract anticholinergic agents, first } \\
\text { generation anti-histamine, second/third generation anti-histamine, short-acting beta-agonist, short- } \\
\text { acting muscarinic antagonist, short-acting beta-agonist plus short-acting muscarinic antagonist, long- } \\
\text { acting beta-agonist plus long-acting beta-agonist, long-acting beta-agonist, xanthine inhibitor, } \\
\text { leukotriene receptor antagonist. }\end{array}$} \\
\hline \multicolumn{7}{|c|}{${ }^{b}$ High blocking affinity is defined as Ki value $<100$ (see Supplementary Table 4). } \\
\hline
\end{tabular}




\section{Discussion}

Although previous studies have shown that the use of antipsychotics at the time of hospital admission increases the risks of asthma-related death and hospital readmission,[13] the association between the use of antipsychotics and severe asthma exacerbation has not been investigated using a nationwide asthma population. The effects of atypical antipsychotics on severe asthma exacerbation have not been examined. In this case-crossover study, we analyzed 18,657 newly diagnosed asthma patients with severe exacerbation leading to ED visits or hospitalization. Using multivariable conditional logistic regression, we found that use of antipsychotics is associated with increase of the risk of severe asthma exacerbation (adjusted OR: 1.27). This result was not confounded by respiratory infection, schizophrenia, use of NSAIDs or non-selective $\beta$-blockers, and different types of inhaled bronchodilator prescription. In the subgroup analysis, use of typical antipsychotics significantly increased the risk of severe asthma exacerbation by $40 \%$. Furthermore, there was a dose-dependent effect of antipsychotics, especially of typical antipsychotics on severe exacerbation of asthma. Analysis for use of atypical antipsychotics did not show an increased risk of severe asthma exacerbation. We thus tentatively conclude that the use of typical antipsychotics is associated with a dose-dependent increased risk of severe asthma exacerbation.

We found that the use of typical antipsychotics led a higher risk of severe asthma exacerbation (adjusted OR: 1.40), whereas the use of atypical antipsychotics did not. This finding is consistent with different adverse events among typical and atypical antipsychotics users. The use of typical antipsychotics is more likely to bring about extrapyramidal symptoms and the use of atypical antipsychotics is often associated with weight gain and metabolic disturbance.[24] A possible explanation for the discordance of side effects between these two groups of drugs is that typical antipsychotics have higher affinity to the dopaminergic receptor and lower affinity to the serotonin receptor compared to those for atypical agents. [10] The higher antipsychotic affinity to specific receptors is associated with a higher risk of different side effects.[25]

We found that simultaneous use of typical and atypical antipsychotics increases risk of severe asthma exacerbation compared to the use of a mono-drug therapy. Combination of two antipsychotics is a widely used strategy for treatment-resistant schizophrenia,[26] and combination of typical and atypical antipsychotics is the most common management in real-world practice.[27] Compare with monotherapy, several studies have reported that combination therapy is associated with increase adverse events and mortality rate.[28-31] Although a recent meta-analysis did not show different risk of serious adverse events between combination antipsychotics and monotherapy, the quality of evidence was very low.[32]

Antipsychotics block the $\beta 2$ adrenergic, M2 muscarinic, D1 and D2 dopaminergic receptors, which are found in human airway smooth muscle with the function of bronchial relaxation. Blocking these bronchodilation receptors increases airway smooth muscle tone and induces muscle spasms, [20-23] causing severe asthma exacerbation. The results stratified by different receptor functions of antipsychotics in our study showed that there were higher risks of severe asthma exacerbation for antipsychotics that function on the M2 muscarinic and D2 dopaminergic receptors. A plausible 
explanation is that most antipsychotics have higher affinities on the M2 muscarinic and D2 dopaminergic receptors than the $\beta 2$ adrenergic receptor (Supplementary Table 4). Blocking the D2 dopaminergic receptor could also induce dystonia of the airway,[33] causing acute exacerbation of an airway disease.

In this study, we found that schizophrenia increased the risk of severe asthma exacerbation. Patients with schizophrenic disorder usually have low adherence to asthma treatment and adopt risky health behaviors such as smoking.[34] Including these patients in the analysis might bias the results. However, the magnitude of the effect was not altered after excluding patients with schizophrenia (Table 3).

Several limitations must be acknowledged in our study. First, the claims data did not include important information such as disease severity and pulmonary function data and we did not take different phenotypes of asthma into consideration. Nevertheless, we adjusted for the use of different inhaled bronchodilators or their combinations, which could be regarded as a surrogate for asthma severity. Consequently, the results were not significantly biased. Second, the diagnoses of asthma and its acute exacerbation should be based on medical history and physical examination instead of ICD codes only. Nevertheless, the accuracy of diagnostic record for asthma in NHIRD had been validated.[16] We further defined newly diagnosed asthma patients as those who had more than one inpatient or two outpatient visits and excluded subjects with the same diagnosis within the prior 2 years. Severe asthma exacerbation was also defined as an acute exacerbation leading to ED visits or hospitalization in combination with the use of short-acting bronchodilators and systemic steroids. Such strict definitions strengthen the validity of our results. Third, adherence to antipsychotics and other medications could not be confirmed using the claims data. Nevertheless, our study used a case-crossover design, in which drug compliance during the case and control period was supposed to be the same. This factor would not significantly affect the results. Forth, we did not include smoking status into the regression model because NHIRD lacks information on smoking status. However, in our case crossover study design, the time interval between the control period and the case period is 60 days, and the smoking status of each enrolled subject may not change in the short period of time. About $70 \%$ of enrolled patient in our study cohort were female. The smoking prevalence of women over 18 years old in Taiwan was $3.9-5.5 \%$ during the enrolled period of this study.[35] In our study, the impact of smoking on asthma severe exacerbation might be small.

\section{Conclusions}

In this nationwide population-based cohort study, patients with asthma showed a dose-dependent increase in the risk of severe asthma exacerbations when receiving antipsychotics. The effect was more significant for patients receiving typical antipsychotics than atypical antipsychotics. Physicians should thus weight the risk and benefit of prescribing high-dose typical antipsychotics for asthma patients.

\section{Abbreviations}


Cl: Confidence interval; CM: Clinical manifestation; DDD: Defined daily dose; ED: Emergency department; ICD-9: International Classification of Diseases, Ninth Revision; NHIRD: National health insurance reimbursement database; OR: odds ratio.

\section{Declarations}

\section{Acknowledgments}

We are grateful to Mrs. Chih-Hui Hsu from the Biostatistics Consulting Center, Clinical Medicine Research Center, National Cheng Kung University Hospital, for providing statistical consulting services. We are also grateful to Health Data Science Center, National Cheng Kung University Hospital for providing administrative and technical support.

\section{Author contributors}

Chin-Wei Kuo and Sheng-Hsiang Lin conceptualized and designed the study. Chin-Wei Kuo, Yu-Fen Shih and Xin-Min Liao assisted with data collection. Chin-Wei Kuo, Szu-Chun Yang and Sheng-Hsiang Lin were in charge of data analysis and interpretation. Chin-Wei Kuo and Szu-Chun Yang drafted the manuscript. Sheng-Hsiang Lin, Szu-Chun Yang and Yu-Fen Shih revised the manuscript critically for important intellectual content. All of the authors approved the final version to be published.

\section{Funding}

This study was funded by grant from the National Cheng Kung University Hospital (NCKUH- 11006009).

\section{Availability of data and materials}

All the data in this study are available to others if the data intend to be used for scholarly purposes such as a systematic review. The data are not publicly available because the use of the National Health Insurance Research Database is limited to research purposes only.

\section{Ethics approval and consent to participate}

The study was approved by the local ethics committee before commencement (The Institutional Review Board of National Cheng Kung University Hospital, IRB number:B-EX-109-026). Informed consent was waived because of the use of de-identified information.

\section{Consent for publication}

Not applicable.

\section{Competing interests}

All authors declare no conflicts of interest. 


\section{References}

1. Papi A, Brightling C, Pedersen SE, Reddel HK: Asthma. Lancet (London, England) 2018, 391(10122):783-800.

2. Collaborators GCRD: Prevalence and attributable health burden of chronic respiratory diseases, 1990-2017: a systematic analysis for the Global Burden of Disease Study 2017. The Lancet Respiratory medicine 2020, 8(6):585-596.

3. Williams SA, Wagner S, Kannan H, Bolge SC: The association between asthma control and health care utilization, work productivity loss and health-related quality of life. Journal of occupational and environmental medicine 2009, 51(7):780-785.

4. Ivanova JI, Bergman R, Birnbaum HG, Colice GL, Silverman RA, McLaurin K: Effect of asthma exacerbations on health care costs among asthmatic patients with moderate and severe persistent asthma. The Journal of allergy and clinical immunology 2012, 129(5):1229-1235.

5. Miller MK, Lee JH, Miller DP, Wenzel SE: Recent asthma exacerbations: a key predictor of future exacerbations. Respiratory medicine 2007, 101(3):481-489.

6. Chien IC, Hsu JH, Bih SH, Lin CH, Chou YJ, Lee CH, Chou P: Prevalence, correlates, and disease patterns of antipsychotic use in Taiwan. Psychiatry and clinical neurosciences 2008, 62(6):677-684.

7. Mark TL: For what diagnoses are psychotropic medications being prescribed?: a nationally representative survey of physicians. CNS drugs 2010, 24(4):319-326.

8. O'Brien PL, Cummings N, Mark TL: Off-Label Prescribing of Psychotropic Medication, 2005-2013: An Examination of Potential Influences. Psychiatric services (Washington, DC) 2017, 68(6):549-558.

9. Carton L, Cottencin O, Lapeyre-Mestre M, Geoffroy PA, Favre J, Simon N, Bordet R, Rolland B: OffLabel Prescribing of Antipsychotics in Adults, Children and Elderly Individuals: A Systematic Review of Recent Prescription Trends. Current pharmaceutical design 2015, 21(23):3280-3297.

10. Miyamoto S, Miyake N, Jarskog LF, Fleischhacker WW, Lieberman JA: Pharmacological treatment of schizophrenia: a critical review of the pharmacology and clinical effects of current and future therapeutic agents. Molecular psychiatry 2012, 17(12):1206-1227.

11. Siafis S, Tzachanis D, Samara M, Papazisis G: Antipsychotic Drugs: From Receptor-binding Profiles to Metabolic Side Effects. Current neuropharmacology 2018, 16(8):1210-1223.

12. Morales DR, Lipworth BJ, Donnan PT, Jackson C, Guthrie B: Respiratory effect of beta-blockers in people with asthma and cardiovascular disease: population-based nested case control study. $B M C$ medicine 2017, 15(1):18.

13. Crane J, Pearce N, Burgess C, Woodman K, Robson B, Beasley R: Markers of risk of asthma death or readmission in the 12 months following a hospital admission for asthma. International journal of epidemiology 1992, 21(4):737-744.

14. Mossman D, Lehrer DS: Conventional and atypical antipsychotics and the evolving standard of care. Psychiatric services (Washington, DC) 2000, 51(12):1528-1535. 
15. Wang JY, Yao TC, Tsai YT, Wu AC, Tsai HJ: Increased Dose and Duration of Statin Use Is Associated with Decreased Asthma-Related Emergency Department Visits and Hospitalizations. The journal of allergy and clinical immunology In practice 2018, 6(5):1588-1595.e1581.

16. Su VY, Yang KY, Yang YH, Tsai YH, Perng DW, Su WJ, Chou KT, Su KC, Yen YF, Chen PC: Use of ICS/LABA Combinations or LAMA Is Associated with a Lower Risk of Acute Exacerbation in Patients with Coexistent COPD and Asthma. The journal of allergy and clinical immunology In practice 2018, 6(6):1927-1935.e1923.

17. Wang MT, Tsai CL, Lin CW, Yeh CB, Wang YH, Lin HL: Association Between Antipsychotic Agents and Risk of Acute Respiratory Failure in Patients With Chronic Obstructive Pulmonary Disease. JAMA psychiatry 2017, 74(3):252-260.

18. Wang MT, Lin CW, Tsai CL, Wang YH, Lai JH, Yeh CB, Huang YL, Hsu YJ: Use of antipsychotics and the risk of acute respiratory failure among adults: A disease risk score-matched nested case-control study. British journal of clinical pharmacology 2020.

19. Leucht S, Samara M, Heres S, Davis JM: Dose Equivalents for Antipsychotic Drugs: The DDD Method. Schizophrenia bulletin 2016, 42 Suppl 1(Suppl 1):S90-94.

20. Kotlikoff MI, Kamm KE: Molecular mechanisms of beta-adrenergic relaxation of airway smooth muscle. Annual review of physiology 1996, 58:115-141.

21. Fryer AD, Jacoby DB: Muscarinic receptors and control of airway smooth muscle. American journal of respiratory and critical care medicine 1998, 158(5 Pt 3):S154-160.

22. Mizuta K, Zhang Y, Xu D, Mizuta F, D'Ovidio F, Masaki E, Emala CW: The dopamine D1 receptor is expressed and facilitates relaxation in airway smooth muscle. Respiratory research 2013, 14(1):89.

23. Mizuta K, Zhang Y, Xu D, Masaki E, Panettieri RA, Jr., Emala CW: The dopamine D(2) receptor is expressed and sensitizes adenylyl cyclase activity in airway smooth muscle. American journal of physiology Lung cellular and molecular physiology 2012, 302(3):L316-324.

24. Mathews M, Muzina DJ: Atypical antipsychotics: new drugs, new challenges. Cleveland Clinic journal of medicine 2007, 74(8):597-606.

25. Olten $\mathrm{B}$, Bloch $\mathrm{MH}$ : Meta regression: Relationship between antipsychotic receptor binding profiles and sideeffects. Progress in neuro-psychopharmacology \& biological psychiatry 2018, 84(Pt A):272-281.

26. Dold M, Leucht S: Pharmacotherapy of treatment-resistant schizophrenia: a clinical perspective. Evidence-based mental health 2014, 17(2):33-37.

27. Gallego JA, Bonetti J, Zhang J, Kane JM, Correll CU: Prevalence and correlates of antipsychotic polypharmacy: a systematic review and meta-regression of global and regional trends from the 1970s to 2009. Schizophrenia research 2012, 138(1):18-28.

28. Gallego JA, Nielsen J, De Hert M, Kane JM, Correll CU: Safety and tolerability of antipsychotic polypharmacy. Expert opinion on drug safety 2012, 11(4):527-542.

29. Mclntyre RS, Jerrell JM: Metabolic and cardiovascular adverse events associated with antipsychotic treatment in children and adolescents. Archives of pediatrics \& adolescent medicine 2008, 
162(10):929-935.

30. Jerrell JM, Mclntyre RS: Adverse events in children and adolescents treated with antipsychotic medications. Human psychopharmacology 2008, 23(4):283-290.

31. Joukamaa M, Heliövaara M, Knekt P, Aromaa A, Raitasalo R, Lehtinen V: Schizophrenia, neuroleptic medication and mortality. The British journal of psychiatry: the journal of mental science 2006, 188:122-127.

32. Ortiz-Orendain J, Castiello-de Obeso S, Colunga-Lozano LE, Hu Y, Maayan N, Adams CE: Antipsychotic combinations for schizophrenia. The Cochrane database of systematic reviews 2017, 6(6):Cd009005.

33. Yaginuma K, Watanabe M, Miyazaki K, Ono A, Murai H, Nodera M, Suzuki Y, Suyama K, Kawasaki Y, Hosoya M: Haloperidol-Induced Dystonia due to Sedation for Upper Gastrointestinal Endoscopy: A Pediatric Case Report. Case reports in emergency medicine 2019, 2019:3591258.

34. Joseph KS: Asthma mortality and antipsychotic or sedative use. What is the link? Drug safety 1997, 16(6):351-354.

35. Chiang $\mathrm{CY}$, Chang HY: A population study on the time trend of cigarette smoking, cessation, and exposure to secondhand smoking from 2001 to 2013 in Taiwan. Popul Health Metr 2016, 14:38.

\section{Figures}


Subjects with newly diagnosed asthma from 2003 to $2016(n=2493305)$

Excluded:

(1) Patients with COPD, chronic bronchiolitis, emphysema, or bronchiectasis $(n=825100)$

(2) Missing age information $(n=970)$

(3) Younger than 18 years $(n=60497)$

(4) Missing sex information $(n=406)$

(5) No asthma-related ED visit or hospitalization ( $n=$ 1384128)

(6) Asthma-related ED visit or hospitalization within 180 days of asthma diagnosis $(n=203547)$

Severe asthma exacerbation resulting in

ED visit or hospitalization

from 2003 to $2016(n=18657)$

b

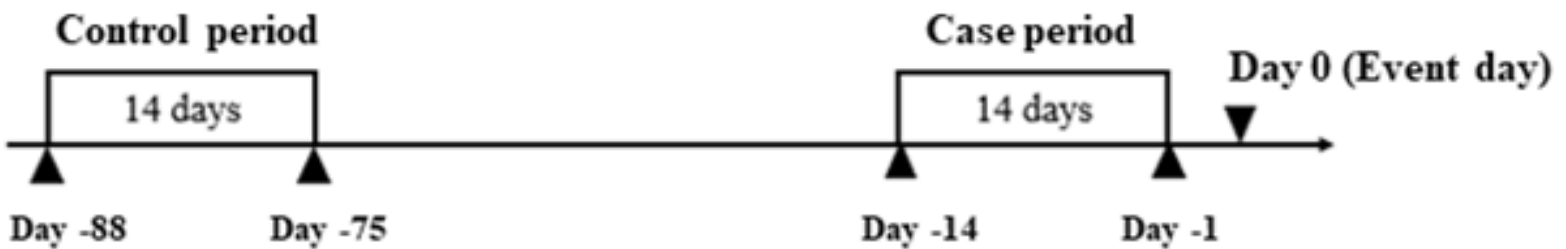

\section{Figure 1}

Algorithm for subject enrollment and timeline for case crossover study. a. Inclusion algorithm for severe asthma exacerbation; b. Timeline for the case and control periods. COPD, chronic obstructive pulmonary disease; ED, emergency department.

\section{Supplementary Files}

This is a list of supplementary files associated with this preprint. Click to download.

- Supplementarydata.docx 